Gynäkologe 2014 • 47:323-324

DOI 10.1007/s00129-013-3257-0

Online publiziert: 20. Mai 2014

c) Springer-Verlag Berlin Heidelberg 2014

\author{
O. Ortmann ${ }^{1} \cdot$ R. Kreienberg ${ }^{2}$ \\ ${ }^{1}$ Klinik für Frauenheilkunde und Geburtshilfe der Universität Regensburg, \\ Caritas-Krankenhaus St. Josef, Regensburg \\ ${ }^{2}$ Landshut
}

\title{
Früherkennungs- und Nachsorgeuntersuchungen
}

diskutiert. Das in Deutschland implementierte nationale Mammographiescreening hat immer noch zu geringe Teilnahmeraten, auch wenn Fortschritte zu verzeichnen sind. A. Scharl gibt in seinem Beitrag eine ausgewogene NutzenRisiko-Abwägung und betont, dass das Mammographiescreening nur nach individueller Aufklärung der Patientin durchgeführt werden sollte. Im Krebsfrüherkennungs- und -registergesetz ist die Einführung eines Zervixkarzinomscreenings vorgesehen. Eine eindeutige Vorgehensweise ist derzeit noch nicht festgelegt. Es existieren auch inhaltlich erhebliche Kontroversen über die exakte Form des Screenings, deren Inhalte und Einladungssysteme. Diese müssen insbesondere vor dem Hintergrund einer bereits seit vielen Jahren implementierten Zervixkarzinomfrüherkennung differenziert betrachtet werden. P. Hillemanns et al. stellen den derzeitigen Kenntnisstand dar und diskutieren verschiedene Szenarien. Das Endometriumkarzinom wird häufig in Frühstadien diagnostiziert. Dazu trägt insbesondere das Frühsymptom Postmenopausenblutung bei. Im Rahmen der derzeit etablierten Krebsfrüherkennungsuntersuchungen ist somit die Frage nach diesem Symptom von essenzieller Bedeutung. Für ein organisiertes Screening liegt selbst bei Hochrisikopopulationen keine ausreichende Evidenz vor. P. Mallmann stellt in seinem Beitrag die aktuelle Datenlage zu der Problematik vor. Seit vielen Jahren wird versucht, verschiedene Strategien zur Früherkennung des Ovarialkarzinoms zu erarbeiten. Geeignete Methoden zur Erreichung dieses Ziels wären gerade bei diesem Malignom von hohem Wert, da es nach wie vor überwiegend in fortgeschrittenen Stadien mit ungünstiger Prognose diagnostiziert wird. Leider haben prospektiv randomisierte Studien zum Ovarialkarzinomscreening bisher keine Verbesserung der krankheitsbedingten Mortalität nachweisen können. Die eingesetzten Methoden waren die Transvaginalsonographie und/oder CA125-Bestimmungen. U. Wagner und K. Baumann kommen nach Darstellung des Kenntnisstandes zu dem Schluss, dass bei fehlendem Effekt der überprüften Methoden auf die Mortalität ein generelles Screening derzeit nicht empfohlen werden kann.

\section{》) Apparative und Labordiagnostik sollten in der Nachsorge gezielt eingesetzt werden}

Nach behandeltem Mammakarzinom bzw. gynäkologischen Malignomen werden derzeit strukturierte Nachsorgeuntersuchungen vorgeschlagen. Diese beinhalten in den ersten drei Jahren relativ hochfrequente Untersuchungsintervalle von 3 Monaten, die tumoradaptiert apparative Untersuchungen einbeziehen. In erster Linie ist die Erkennung von Lokalrezidiven Ziel der Nachsorge. Für alle gynäkologischen Malignome und das Mammakarzinom hat sich bisher kein Nutzen für die Suche nach Fernmetastasen beweisen lassen. Daher sind umfangreiche apparative Untersuchungen oder Tumormarkerbestimmungen, die dieses Ziel verfol-
Nach wie vor wird das Mammographiescreening international kontrovers 
gen, nicht zu empfehlen. Sie sollten nur bei Vorhandensein von Symptomen zielgerichtet eingesetzt werden.

Von besonderer Bedeutung ist die Überprüfung der Lebensqualität der Patientinnen, die psychosoziale Beratung sowie Empfehlungen allgemeiner gesundheitsfördernder Maßnahmen. Gerade bei den Genitalmalignomen spielt auch die Sexualberatung eine wichtige Rolle. Für die Zukunft ist zu fordern, dass im Rahmen der Nachsorgeuntersuchung und der damit verbundenen Beratung oben angegebene Aspekte neben der Rezidivdiagnostik besondere Beachtung finden. In den Beiträgen von $S$. Buchholz und O. Ortmann sowie M. Bossart und A. Hasenburg wird die heute als sinnvoll angesehene Vorgehensweise umfassend erläutert. Wir wünschen den Lesern bei der Lektüre dieser Kapitel viel Freude und hoffen, dass die Informationen für die tägliche Praxis von Nutzen sind.

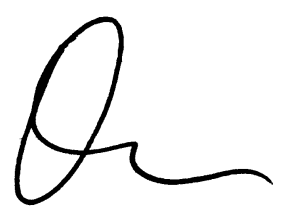

Prof. Dr. Olaf Ortmann

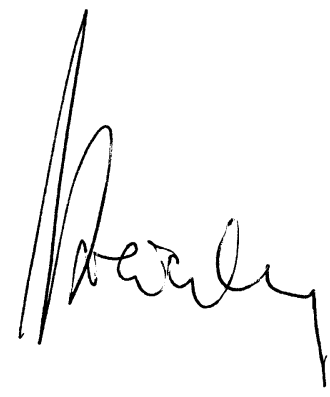

Prof. Dr. Rolf Kreienberg

\section{Korrespondenzadresse}

Prof. Dr. 0. Ortmann

Klinik für Frauenheilkunde und Geburtshilfe der Universität Regensburg, Caritas-Krankenhaus St. Josef Landshuter Str. 65, 93053 Regensburg olaf.ortmann@klinik.uni-regensburg.de

Interessenkonflikt. O. Ortmann gibt an, dass kein Interessenkonflikt besteht.

\section{Neu: Interdisziplinäre Fortbildung der Deutschen Akademie für Senologie}

Die Behandlung von Brustkrebs verlangt von Medizinern vielfältige Kompetenzen - neben der eigenen fachärztlichen Expertise sind für die Zusammenarbeit in interdisziplinären Behandlungsteams auch Kenntnisse der anderen beteiligten Fachdisziplinen nötig. Um diese Fähigkeiten zu stärken, startet die Deutsche Akademie für Senologie (DAS) ein interdisziplinäres, strukturiertes Fortbildungsprogramm. Das modular aufgebaute Programm bietet Fortbildungsmodule für sieben Fachdisziplinen: Senologie für Pathologen, Radiologen, Gynäkologen, Radioonkologen, Chirurgen, Plastische Chirurgen und Internisten. Mit dem Besuch von sieben Theoriemodulen und einer Praxiswoche (5 Arbeitstage) kann so das „Zertifikat der Deutschen Akademie für Senologie" erworben werden. Die Praxiswoche wird in einem namhaften zertifizierten Brustzentrum absolviert, um die theoretischen Module in der Praxis zu vertiefen und wesentliche Fragen mit allen beteiligten Kooperationspartnern zu diskutieren.

Die ersten Theoriemodule finden auf der diesjährigen Jahrestagung der Deutschen Gesellschaft für Senologie vom 19.-21. Juni 2014 in Berlin statt. Als zusätzliche Qualifikation darf die Fortbildung nach oder im Rahmen der Facharztausbildung nach mindestens drei Jahren Weiterbildungszeit begonnen werden. Die Kombination aus Theorie- und Praxismodulen macht die Fortbildung zu einem interdisziplinären Komplettangebot. Das anschließend verliehene Zertifikat ist für fünf Jahre gültig. Voraussetzung ist die Mitgliedschaft in der Deutschen Gesellschaft für Senologie (DGS). Fachliche Leiter für die einzelnen Module sichern im Kontakt mit den Referenten die Qualität.

Quelle: Deutsche Gesellschaft für Senologie, www.senologie.org 